\title{
Clonal seeds in hybrid rice using CRISPR/Cas9
}

3 Chun Wang ${ }^{1}$, Qing Liu ${ }^{1}$, Yi Shen ${ }^{2}$, Yufeng Hua ${ }^{1}$, Junjie Wang ${ }^{1}$, Jianrong Lin ${ }^{1}$,

$4 \quad$ Mingguo $\mathrm{Wu}^{1}$, Tingting Sun ${ }^{1}$, Zhukuan Cheng $^{2}$, Raphael Mercier ${ }^{3}$, Kejian Wang $^{1}$

$6{ }^{1}$ State Key Laboratory of Rice Biology, China National Rice Research Institute,

7 Chinese Academy of Agricultural Sciences, Hangzhou 310006, China.

$8 \quad{ }^{2}$ State Key Laboratory of Plant Genomics, Institute of Genetics and Developmental

9 Biology, Chinese Academy of Sciences, Beijing 100101, China.

$10{ }^{3}$ Institut Jean-Pierre Bourgin, INRA, AgroParisTech, CNRS, Université Paris-Saclay, 11 RD10, 78000 Versailles, France.

12 Correspondence should be addressed to K.W. (wangkejian@caas.cn) 
Heterosis, the observation that first generation hybrids outcompete the parental lines, is widely used in increasing the productivity and yield of agricultural crops $^{1,2}$. However, heterosis is lost in the following generations because of genetic segregation. In addition, the high cost of hybrid seed production hinders the application of heterosis in many crops. Clonal reproduction through seeds could be revolutionary for agriculture by allowing self-propagation of $F_{1}$ hybrids ${ }^{3,4}$. Here we show that heterozygosity of $F_{1}$ hybrid rice can be fixed and thus propagated without additional crossing. First, we showed that multiplex editing of three key meiotic genes ${ }^{5,6}$ in hybrid rice leads to the production of clonal diploid gametes and tetraploid seeds. Next, editing of the MATRILINEAL (MTL) gene that involved in fertilization ${ }^{7,8}$ results in the induction of haploid seeds in hybrid rice. By simultaneous editing of these four endogenous genes in hybrid rice using the CRISPR/Cas9 system, we obtained in one generation plants able to propagate clonally through seeds. This opens the possibility to fix heterozygosity of hybrid varieties in food crops.

Heterosis (also known as hybrid vigor) is a phenomenon whereby hybrid offspring of genetically diverse individuals display increased vigor relative to their homozygous parents. Heterosis has been widely applied in agriculture to dramatically improve the production and to broaden adaptability of crops $^{1,2}$. However, the essential process of hybrid seed production increases the seed cost and even prohibits its application in many crops. It has been proposed to fix the heterosis of hybrid crop by introduction of apomixis ${ }^{3}$. Apomixis is an asexual reproductive strategy where the 
offspring were generated through seeds, but without meiosis and fertilization. Although it has been described in many flowering plant taxa ${ }^{9}$, apomixis has not been reported in major crops. Previously, it was revealed that combined mutations of three genes that affect key meiotic processes created a genotype called MiMe (Mitosis instead of Meiosis) in which meiosis is totally replaced by mitotic-like division, leading to the production of male and female clonal diploid gametes in Arabidopsis and rice ${ }^{5,6}$. However, the self-fertilization of MiMe resulted in doubling of ploidy at each generation. By crossing Arabidopsis MiMe with CenH3-mediated chromosome elimination line, clonal diploid offspring were obtained ${ }^{4}$. However, the system still relies on the crossing between different plants and the CENH3-mediated chromosome elimination appeared to be difficult to transfer to other species ${ }^{10}$. Therefore, further work is required to achieve the aim of heterosis fixation in self-fertilized hybrids.

Firstly, to test the feasibility of MiMe technology in hybrid rice varieties, we performed experiments on Chunyou84 (CY84), an elite inter-subspecific hybrid rice from a cross between the maternal Chunjiang 16A (16A), a japonica male sterile line, and the paternal C84, an indica-japonica intermediate type line (Extended Data Fig.1). To ensure rapid generation of MiMe in the hybrid CY84 background, we simultaneously edited the REC8, PAIRI and OSD1 genes using our previously developed multiplex CRISPR/Cas9 system $^{11}$ (Fig. 1a). In the primary transformed plants, 7 of 32 plants were identified as frameshift triple mutants, and three of them were analyzed (Extended Data Fig.2). The triple mutant (MiMe) could not be distinguished from the wild-type CY84 based on its growth or morphology (Extended 
Data Fig.3). To test whether the meiosis was turned into a mitotic-like division, we investigated the male meiotic chromosome behavior in both wild type and MiMe. In the wild-type CY84 (Extended Data Fig.4a-f), 12 bivalents were scattered at diakinesis and aligned along the equatorial plate at metaphase I. The 12 pairs of homologous chromosomes separated at anaphase I and produced tetrad spores after the second meiotic division. In MiMe (Extended Data Fig.4g-i), 24 univalents were found in diakinesis and aligned at metaphase I. In anaphase I, 24 pairs of chromatids segregated into two groups and produced dyads of spores, suggesting that the meiosis has been turned into a mitotic-like division. We next examined the ploidy of spores of MiMe by performing fluorescent in situ hybridization (FISH) analyses using a $5 \mathrm{~S}$ rDNA-specific probe, which identifies chromosome 11 of rice. Only one signal was observed in CY84 spores $(n=30)$, while two signals were constantly observed in MiMe spores (n=40, Fig. 1b), showing that diploid gametes were generated in MiMe. We also investigated the fertility of MiMe mutant and found that the panicle seed setting rate in MiMe was $\sim 81.2 \%(\mathrm{n}=4043)$, which is comparable to that of wild type $(\sim 79.1 \%, n=3876)$, (Fig.1c, Table 1), suggesting that simultaneously editing of these three genes do not obviously affect fertility in this hybrid variety. The ploidy of the progeny of MiMe plant was investigated by flow cytometry and all $(n=123)$ were found to be tetraploid plants (Fig.1d, Table 1). Further, we found that these progenies $(n=123)$ retained completely the heterozygosity of their parent CY84 for 10 tested Insertion-deletion (Indel) makers (Fig.1e). And these progenies of MiMe displayed reduced fertility, increased grain size and elongated awn length compared to wild type, 
all of which being typical characteristics of tetraploid rice (Fig.1f). These results show that the MiMe phenotype can be rapidly introduced into hybrid rice varieties using CRISPR/Cas9 genome editing technique.

MiMe clonal gametes participate in normal self-fertilization, giving rise to progeny with doubled ploidy. This ploidy doubling must be prevented to achieve apoximis. Recently, it was reported that the MATRILINEAL (MTL) gene, a sperm-specific phospholipase, triggers haploid induction in maize $\mathrm{e}^{7,8}$. To test whether the homologous gene could be manipulated to induce haploid in self-fertilized hybrid rice, we edited the MTL gene in CY84 (Fig. 2a). 11 of 32 transformed plants were identified as frameshift mutants, and three of them were analyzed (Extended Data Fig.5). The $m t l$ mutants showed normal vegetative growth (Extended Data Fig.3), but the seed-setting rates significantly reduced to $\sim 11.5 \%$ ( $\mathrm{n}=5180$, Fig. $2 \mathrm{~b}$, Table 1 ). 12 Indel markers ( 1 per chromosome) that were polymorphic between the two parents were used to determine the genotype of the progenies of $m t l$ plants (Extended Data Table1). In the wild-type CY84 progeny, no plants homozygous at all markers were found ( $\mathrm{n}=220$, Table 1). In contrast, 11 plants among $248 \mathrm{mtl}$ progenies appeared to be homozygous for all markers (Fig. 2c, Table 1). Flow cytometry results showed that 9 of these plants were indeed haploid, while 2 were diploid, presumably resulting from spontaneous doubling of haploid embryos (Fig. 2d, Table 1). To further classify the genotype of those identified plants, the whole genomes of 2 haploids, 2 doubled haploids of $m t l$ progenies, and 2 offspring plants of wild-type CY84 were resequenced with a depth of 30 -fold. A total of 78,909 single nucleotide polymorphisms (SNPs) 
102

103

that differed between two parents were screened out for detailed genotype analysis.

Whole genome sequencing revealed that the haploids and doubled haploids were homozygous at all loci (Fig. 2e), and recombinant compared to the parental genome, suggesting that they are respectively derived from a single gamete. The haploid plants showed reduced plant height, decreased glume size and loss of fertility, while the doubled haploid plant displayed normal vegetative and reproductive growth (Fig. 2f). The results demonstrated that haploid plants can be generated by self-fertilization of hybrid varieties.

Since turning meiosis into mitosis and paternal genome elimination is possible in self-fertilized hybrid rice, we next test the possibility of inducing heterozygosity fixation without additional crossing in hybrid rice by simultaneously editing four genes, namely OSD1, PAIR1, REC8 and MTL in CY84 (Fig. 3a-b). Among 22 transgenic plants, three were identified by DNA sequencing as osd1/pair $1 / \mathrm{rec} 8 / \mathrm{mtl}$ quadruple mutants (namely Fix, Fixation of hybrids) and used for further analysis (Extended Data Fig.6). The Fix mutants grew normally during the vegetative stage (Fig. 3c). During reproductive stage, the male meiotic chromosome behavior was investigated and found to be indistinguishable from that of MiMe (Extended Data Fig.4j-1). The panicle seed setting percentage was found to be $\sim 4.5 \%(\mathrm{n}=5850)$ (Table 1, Fig. 3c), which is slightly lower than that of the $m t l$ mutant. In the progeny seedlings, the ploidy was investigated using flow cytometry. Among 145 progeny of Fix mutants, 136 were identified as tetraploid and 9 as diploid (Fig. 3d, Table 1). To investigate whether the heterozygosity was fixed in these diploid offspring, the 
124

125

genomes of 2 diploid and 2 tetraploid offspring plants of Fix were resequenced with an average of $30 \times$ coverage. Bioinformatic analysis revealed that all the 78,909 SNPs were heterozygous in both these diploid and tetraploid progeny plants, and were thus genetically identical to the hybrid rice CY84 (Fig. 3e). Finally, we investigated the phenotype of the potential clonal plants of Fix. All these 9 diploid plants displayed normal glume size and awn length, and showed a reduced seed setting $(\sim 10 \%$, n=2726), which were similar to their parent Fix plants (Fig. 3f). Taken together, the diploid progeny of Fix plant displayed the same ploidy, the same heterozygous genotype, and the similar phenotype with the parent Fix plants, implying that Fix is able to produce clonal seeds and fix the heterozygosity of hybrid rice.

Our findings revealed that hybrids can be self-propagated through seeds by targeted editing of four endogenous genes in rice hybrid varieties. Simultaneous editing of REC8, PAIR1 and OSD1 genes does not have obvious adverse effects on the growth and reproduction of the hybrid. On contrast, the MTL gene used to induce paternal genome elimination has impacts on rice fertility and is not fully penetrant; further work is thus required to allow this technology to reach the rice fields. However, the findings in this study revealed a strategy to fix heterozygosity in rice. Considering the establishment of multiplex genome editing technology in many other crops along with the conservation of these four genes, the strategy might extend heterosis application in agriculture. 


\section{Methods}

Plasmid construction. The plasmids expressing the CRISPR/Cas9 system were constructed via the isocaudamer ligation method, as previously described ${ }^{11}$. The modified single guide RNAs (sgRNAs) scaffold and ACTIN1 promoter-driven Cas9 were used to increase the mutation rate in this study ${ }^{12}$. Briefly, the double-stranded overhangs of target oligoes (listed in Extended Data Table1) were ligated into the SK-sgRNA vectors digested with AarI. Then the sgRNAs of OSD1 (digested with KpnI and SalI), PAIRI (digested with $X h o \mathrm{I}$ and BglII) and REC8 (digested with BamHI and NheI) were assembled into one pC1300-ACT:Cas9 binary vector (digested with KpnI and XbaI) using T4 ligase to obtain the vector pC1300-ACT:Cas9-sgRNA ${ }^{\text {OSD1 }}$-sgRNA ${ }^{\text {PAIR1 }}$-sgRNA ${ }^{\text {REC8 }}$ for generation of MiMe. The sgRNA of MTL (digested with KpnI and NheI) was assembled into pC1300-ACT:Cas9 binary vector (digested with KpnI and $X b a \mathrm{I}$ ) to obtain the vector pC1300-ACT:Cas9-sgRNA ${ }^{\text {MTL }}$ for generation of $m t l$. The sgRNA of MTL (digested with $\quad$ KpnI NheI) was assembled into pC1300-ACT:Cas9-sgRNA OSD1-sgRNA ${ }^{\text {PAIR1-sgRNA }}{ }^{\text {REC8 }}$ vector (digested with $K p n I$ and $X b a \mathrm{I})$ to obtain the vector pC1300-ACT:Cas9-sgRNA ${ }^{\text {OSD1 }}$-sgRNA ${ }^{\text {PAIR1 }}$ $\operatorname{sgRNA}^{\mathrm{REC} 8}$-sgRNA ${ }^{\mathrm{MTL}}$ for generation of Fix.

Rice transformation and growth conditions. The hybrid rice Chunyou 84 (CY84) was used as the host variety in this study. The generation of transgenic rice, by Agrobacterium-mediated transformation with the strain EHA105, was performed by the Biogle company (Hangzhou, China). 

winter of 2017.

Detection of genome modifications. Genomic DNA was extracted from approximately $100 \mathrm{mg}$ of rice leaf tissue via the CTAB method. PCR was conducted with KOD FX DNA Polymerase (Toyobo, Osaka, Japan) to amplify the genomic regions surrounding the target sites. The primers are listed in Extended Data Table1. The fragments were sequenced by the Sanger method and decoded by the degenerate sequence decoding method ${ }^{13}$.

177 Cytological analyses. Young panicles of meiosis stage were harvested and fixed in 178 Carnoy's solution (ethanol:glacial acetic, 3:1). Microsporocytes undergoing meiosis were squashed in an acetocarmine solution. Slides were frozen in liquid nitrogen and the coverslips were removed with a blade quickly. Chromosomes were counterstained with 4',6-diamidinophenylindole (DAPI) in an antifade solution (Vector Laboratories, Burlingame, CA). Microscopy was conducted using an Olympus BX61 fluorescence microscope with a microCCD camera. previously ${ }^{14}$. The plasmid pTa794 was used as FISH probe to quantify the $5 \mathrm{~S}$ rDNA.

Fluorescence in situ hybridizaiton (FISH) analysis was conducted as described Genotyping with Indel Markers. Insertion-deletion (Indel) markers to distinguish genotypes of heterozygote and homozygote were designed based on the 
189

190

191

192

193

194

195

Table1. The genotyping was performed by normal PCR program using $2 \times$ Taq Master Mix (Novoprotein Scientific, China), and the PCR products were detected using 5\% agarose gels.

Flow cytometry determination of DNA content in leaf cell nuclei. The ploidy of leaf cell was determined by estimating nuclear DNA content using flow cytometry. All procedures were done at $4{ }^{\circ} \mathrm{C}$ or on ice. Approximately $\sim 2 \mathrm{~cm}^{2}$ of leaf tissue was chopped using a new razor blade for 2 to 3 minutes in $1 \mathrm{ml} \mathrm{LB} 01$ Buffer (15 mM Tris, $2 \mathrm{mM} \mathrm{Na} 2$ EDTA, $0.5 \mathrm{mM}$ spermine tetrahydrochloride, $80 \mathrm{mM} \mathrm{KCl}, 20 \mathrm{mM} \mathrm{NaCl}$, $0.1 \%$ Triton $\mathrm{X}-100,15 \mathrm{mM} \beta$-mercaptoethanol, $\mathrm{pH} 7.5$, filter through a $0.22 \mu \mathrm{m}$ filter). The homogenate was filtered through the 40- $\mu$ m nylon filter followed by centrifugation $(1200 \times \mathrm{rpm}, 5 \mathrm{~min})$ to collect the nuclei. The supernatant was discarded and the pellet was resuspended with $450 \mu \mathrm{L}$ of fresh LB01 Buffer, then 25 $\mu \mathrm{l}$ of $1 \mathrm{mg} / \mathrm{ml}$ propidium iodide (PI, Sigma P4170) and $25 \mu \mathrm{lof} 1 \mathrm{mg} / \mathrm{ml}$ DNase-free RNase A (Sigma V900498) were added to stain the DNA. The stained samples were incubated on ice in darkness for 10 minutes prior to analysis. The samples were analyzed using BD Accuri C6 flow cytometer, with the laser illumination at $552 \mathrm{~nm}$ and 610/20 nm filter. The gating strategy was provided in Supplementary Information. Samples with the same result of CY84 were deemed as diploids, which the first peak of relative fluorescence at $\sim 100(\mathrm{x} 10,000)$. And the samples with the first peak of relative fluorescence at $\sim 50(\mathrm{x} 10,000)$ were deemed as haploids, while samples with the first peak of relative fluorescence at $\sim 200(\mathrm{x} 10,000)$ were deemed as tetraploids.

Whole genome re-sequencing and genotype calling. The 150-bp paired-end reads 
211

were generated by Illumina Hiseq2500, covering approximately an average depth of $30 \times$ for each sample. The short-read sequence data have been deposited in the NCBI Sequence Read Archive (SRP149641, SRP149677). The raw paired-end reads were first filtered into clean data using NGSQCtookit v2.3.3 ${ }^{15}$. The cutoff value for PHRED quality score was set to 30 . Clean reads of each accession were aligned against the rice reference genome (IRGSP 1.0) using the software SOAPaligner ( soap version 2.21) ${ }^{16}$ with the parameters of ' $-\mathrm{m} 200$, -x 1000, -135 , -s 42, -v 5' and '-p 8'. To get high-quality SNPs, reads that could be mapped to different genomic positions were excluded by SOAPsnp ${ }^{17}$. Uniquely mapped single-end and paired-end results were used in the SNP calling. Genotype calling was carried out in the whole genome region using these SNPs which are heterozygous in the parent. The window size (the number of $\mathrm{n}$ consecutive SNPs in a window) was $0.1 \mathrm{~K}$. And the recombination map was constructed for each chromosome.

Data availability. Whole genome sequencing data are deposited in the NCBI Sequence Read Archive (SRP149641, SRP149677). Patent applications have been filed relating to work in this manuscript.

Schnable, P. S. \& Springer, N. M. Progress toward understanding heterosis in crop plants. Annu Rev Plant Biol 64, 71-88 (2013).

2 Birchler, J. A., Auger, D. L. \& Riddle, N. C. In search of the molecular basis of heterosis. Plant Cell 15, 2236-2239 (2003).

3 Spillane, C., Curtis, M. D. \& Grossniklaus, U. Apomixis technology development-virgin births in farmers' fields? Nat Biotechnol 22, 687-691 (2004).

4 Marimuthu, M. P. A. et al. Synthetic clonal reproduction through seeds. Science 331, 876 
d'Erfurth, I. et al. Turning meiosis into mitosis. PLoS Biology 7, e1000124 (2009). haploid induction. Nature 542, 105-109 (2017). possible cause of maize haploid induction. Nat Commun 8, 991 (2017). Fixation of Phenotypes in Hybrid Plants. Curr Biol 26, 331-337 (2016). induces haploid plants. Proc Natl Acad Sci U S A 112, 11211-11216 (2015). Multiplex Genome Editing in Rice. Journal of genetics and genomics 42, 703-706 (2015). CRISPR-Cas9-VQR precise genome editing in rice. Plant Biotechnology Journal 16, 292-297 (2018). Products. Mol Plant 8, 1285-1287 (2015).

Zhang, W. et al. The transcribed 165-bp CentO satellite is the major functional centromeric element in the wild rice species Oryza punctata. Plant Physiol 139, 306-315 (2005). sequencing data. PLoS One 7, e30619 (2012). 25, 1966-1967 (2009). Res 19, 1124-1132 (2009). 
265 Acknowledgements This research was supported by the Agricultural Science and

266 Technology Innovation Program of Chinese Academy of Agricultural Sciences, and

267 the National Natural Science Foundation of China (No. 31401363).

268 Author Contributions C.W., and K.W. conceived and designed the study. C.W., Y.S.,

269 and Z.C. performed the lab experiments. Q.L., and T.S. conducted the computational

270 analyses. Y.H., and J.W. carried out the field experiments. J.L., and M.W. provided the

271 rice varieties and helped with the field management. C.W., R.M., and K.W. wrote the

272 manuscript.

273 
Table1 Ploidy analysis of the progeny of CY84, MiMe, mtl and Fix lines

\begin{tabular}{|c|c|c|c|c|c|c|}
\hline & Line & Seed setting percentage & $\begin{array}{c}\text { Progeny } \\
\text { tested }\end{array}$ & $\begin{array}{c}\text { Haploid+DH } \\
(\%)\end{array}$ & $\begin{array}{c}\text { Diploid } \\
(\%)\end{array}$ & $\begin{array}{c}\text { Tetraploid } \\
(\%) \\
\end{array}$ \\
\hline \multirow{3}{*}{ CY84 } & $\# 1$ & $77.2 \%(1151 / 1490)$ & 65 & 0 & 65 & 0 \\
\hline & $\# 2$ & $81.3 \%(951 / 1170)$ & 73 & 0 & 73 & 0 \\
\hline & $\# 3$ & $79.1 \%(962 / 1216)$ & 82 & 0 & 82 & 0 \\
\hline \multirow{3}{*}{ MiMe } & $\# 1$ & $81.9 \%(1178 / 1439)$ & 35 & 0 & 0 & $35(100 \%)$ \\
\hline & $\# 2$ & $79.2 \%(877 / 1108)$ & 43 & 0 & 0 & $43(100 \%)$ \\
\hline & $\# 3$ & $82.1 \%(1228 / 1496)$ & 45 & 0 & 0 & $45(100 \%)$ \\
\hline \multirow{3}{*}{$m t l$} & $\# 1$ & $9.1 \%(101 / 1103)$ & 77 & $6+0(7.8 \%)$ & 71 & 0 \\
\hline & $\# 2$ & $13.6 \%(217 / 1601)$ & 90 & $2+1(3.3 \%)$ & 87 & 0 \\
\hline & $\# 3$ & $11.3 \%(280 / 2476)$ & 81 & $1+1(2.5 \%)$ & 79 & 0 \\
\hline \multirow{3}{*}{ Fix } & $\# 1$ & $3.7 \%(63 / 1725)$ & 39 & 0 & $2(5.1 \%)$ & 37 \\
\hline & $\# 2$ & $5.2 \%(124 / 2373)$ & 64 & 0 & $3(4.7 \%)$ & 61 \\
\hline & $\# 3$ & $4.3 \%(76 / 1752)$ & 42 & 0 & $4(9.5 \%)$ & 38 \\
\hline
\end{tabular}


a

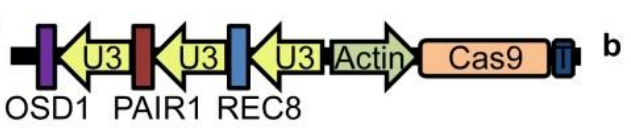

OSD1 Os02g37850

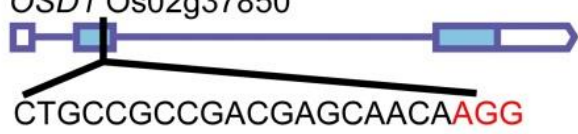

PAIR1 Os03g01590

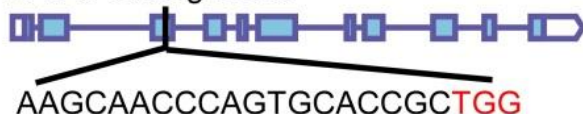

REC8 Os05g50410
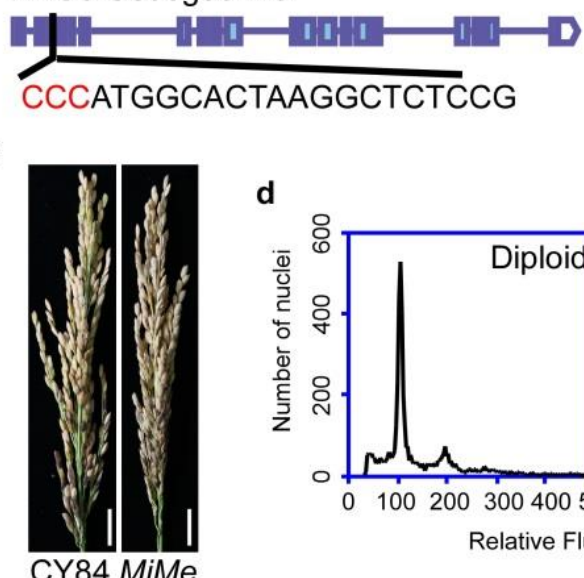

d

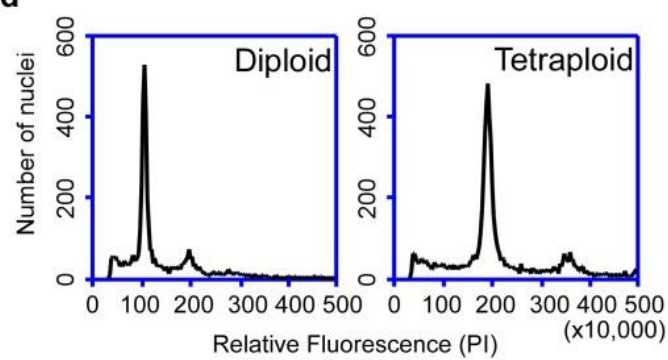

e

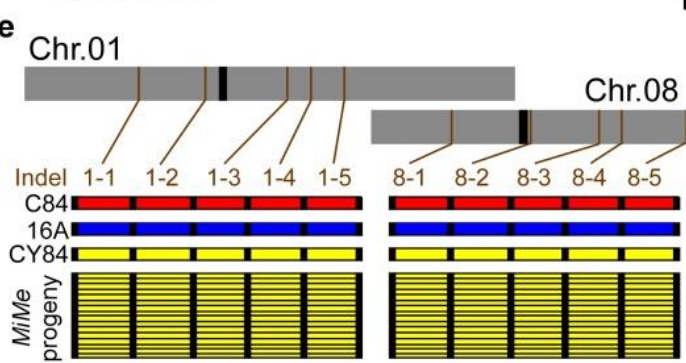

Figure 1|Turning meiosis into mitosis in hybrid rice variety Chunyou84 (CY84).

a, Schematic diagram of the structure of CRISPR/Cas9 vector targeting OSD1, PAIR1 and REC8.

b, The chromosomes

of CY84 and MiMe were probed by digoxige-nin-16-dUTP-labled 5S rDNA (red signal, indicated with white arrow) in spores, showing one signal in wild-type CY84 and two signals in MiMe. Scale bars, 5 $\mu \mathrm{m}$. c, Panicles of wild-type CY84 and MiMe. The fertility of MiMe is as high as that of wild-type CY84. Scale bars, $2 \mathrm{~cm}$. d, Ploidy analysis of CY84 (left) and the 
285 progeny of MiMe (right) by flow cytometry, which is found to be diploid and 286 tetraploid, respectively (Table 1). e, Genotype analysis of the paternal C84, maternal 287 Chunjiang 16A (16A), hybrid variety Chunyou84 (CY84) and the progeny siblings of 288 MiMe. 10 Indel markers distributed on chromosomes 1 and 8 were used to identify the 289 genotype of the offspring of MiMe. Positions of markers (brown) and centromeres 290 (black) are indicated along the chromosomes. For each marker, plants carrying the 291 C84 allele are in red, plants carrying the 16A allele are in blue, while plants with both $292 \mathrm{C} 84$ and 16A alleles appear in yellow. Each row represents one plant, and each 293 column indicates a locus. f, Panicles and grain shape of CY84 and the progeny of 294 MiMe. The progeny of MiMe displayed reduced fertility, increased glume size and 295 elongated awn length. Scale bars, $2 \mathrm{~cm}$. 
297 b

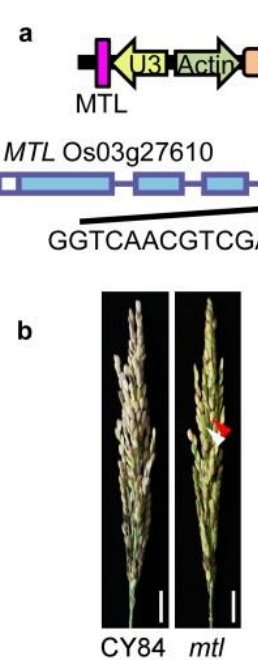

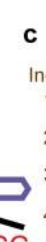

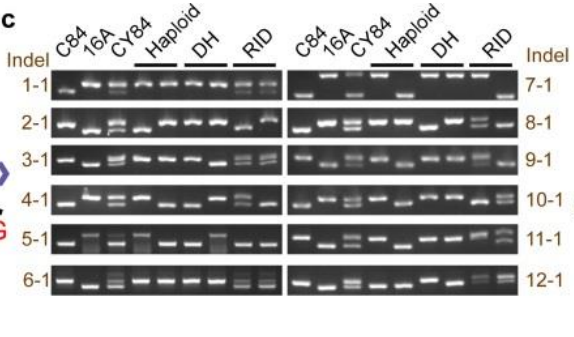

d

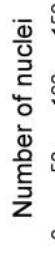

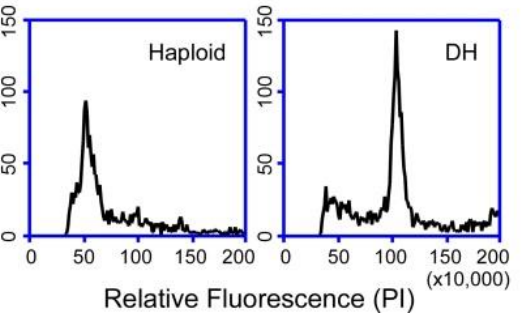
$m t l$ progeny, including RID, haploid and DH plants. Scale bars, $2 \mathrm{~cm}$.

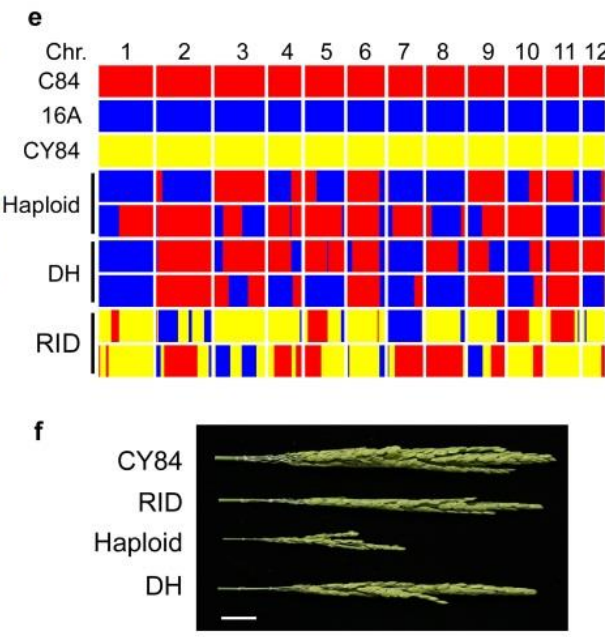

Figure $2 \mid$ Generation of haploid inducer line by editing the $M T L$ gene in hybrid

rice variety CY84. a, Schematic diagram of the structure of CRISPR/Cas9 vector

targeting MTL. b, Panicles of the WT and $m t l$ in CY84 background. The fertility was

decreased in $m t l$, white arrow indicates aborted seed, and red arrow shows fertile seed.

Scale bars, $2 \mathrm{~cm}$. c, Determination of the genotype of haploids, doubled haploids (DH)

and recombinant inbred diploids (RID) using 12 Indel markers (1 per chromosome).

Plants homozygous at all markers in the progeny siblings of $m t l$ were identified as

haploid or DH. d, Ploidy analysis of the haploid and DH by flow cytometry (Table 1).

e, Whole genome sequencing of the haploid, DH and RID plants. 12 blocks represent

12 chromosomes. The SNPs of C84 allele are in red, the SNPs of 16A allele are in

blue, and co-existence of both alleles are in yellow. f, Panicles of wild-type CY84 and 
a

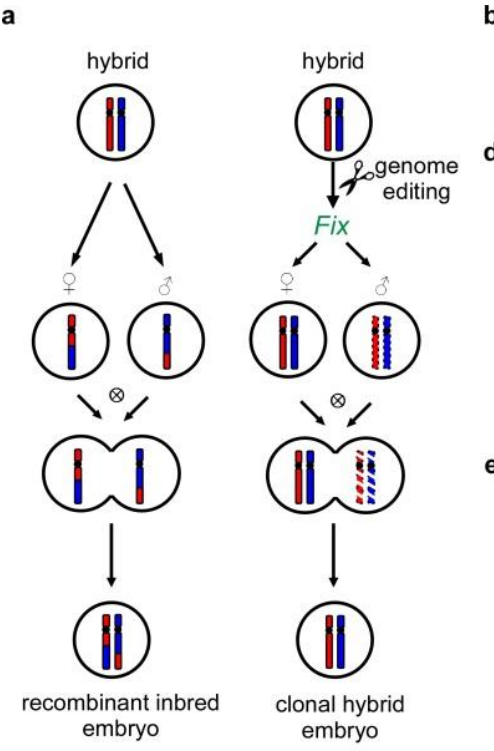

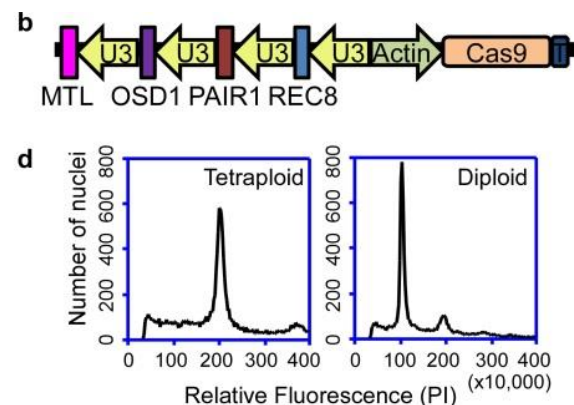

e

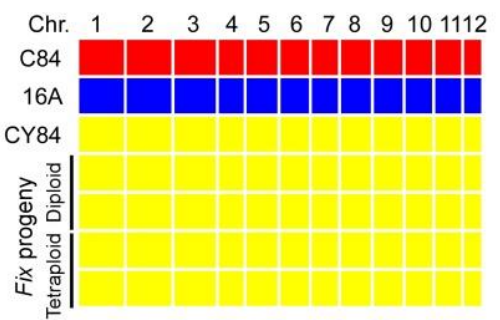

Figure 3 |Fixation of rice heterozygosity by multiplex gene editing in hybrid rice

variety CY84. a, The model of fixation of heterozygosity of hybrid. In normal sexual reproduction (left), recombinant inbred embryos are generated by fusion of recombined haploid gametes. The clonal reproduction strategy (right) is based on two components: meiosis is turned into mitosis to produce clonal diploid gametes (MiMe), and the genome of male gamete is eliminated by knocking out the MTL gene. The progeny of self-fertilized Fix is genetically identical to the hybrid parent. b, Schematic diagram of the structure of CRISPR/Cas9 vector simultaneously targeting OSD1, PAIR1, REC8 and MTL. c, Comparison of the morphology and panicles of CY84 and Fix (osdl pairl rec8 $\mathrm{mtl}$ ). The fertility was decreased in Fix. An aborted seed is indicated with white arrow, and a normally developed seed is indicated with red arrow. Scale bars, $5 \mathrm{~cm}$. d, Ploidy analysis of the progeny of Fix by flow cytometry, including tetraploid (left) and diploid (right), respectively. e, Whole genome sequencing of the diploid and tetraploid progenies of Fix. The SNPs of C84 
326 allele are in red, the SNPs of 16A allele are in blue, and co-existence of both alleles

327 are in yellow. 12 blocks represent 12 chromosomes. The diploid and tetraploid

328 progenies of Fix are heterozygous, identical to CY84. f, Comparison the morphology

329 and panicles of wild-type CY84 and the diploid progeny of Fix. Both plants were

330 grown in the glasshouse. The clonal Fix displayed normal growth except the reduced

331 fertility, which is similar to that of parent Fix plant. Scale bars, $5 \mathrm{~cm}$. 